\title{
Research on Simulation Analysis of Boiler Furnace Leakage Sound Propagation Simulation
}

\author{
Peng Tong \\ Department of Power Engineering \\ Baoding Technical College of Electric Power \\ Baoding, China \\ E-mail: tptong@163.com
}

\author{
Yongqiang $\mathrm{Wu}$ \\ Science Information Center \\ Baoding Technical College of Electric Power \\ Baoding, China \\ E-mail: wuyongqiang@163.com
}

\begin{abstract}
Based on the acoustic field distribution and directivity characteristics when leakage happens in the boiler furnace, the propagation model has been firstly analyzed theoretically and simulated numerically in this paper. Then a shell model has been established to make the sound field simulation and line tracking. Lastly, the variation of sound pressure level has been studied to prove the attenuation law.
\end{abstract}

Keywords- Boiler; Furnace; Leakage Sound; Propagation; Simulation

\section{INTRODUCTION}

It is necessary to study and analyse the acoustic field distribution and transmission law when the leakage happens because they vary greatly in different environments or media. Radiation of source in free space expands gradually in the form of spherical wave, whose amplitude reduces with the increase of distance. While radiation of source must be affected and restricted by such factors as burning, pulverized coal movement or tube array in the boiler furnace, which is approximately confined space. The process can be simplified in Fig. 1.

Based on the theoretical analysis and numerical simulation made to acoustic field distribution and directivity characteristics in the reference [1-5], the far field sound distribution characteristics show similarity in the surface perpendicular to tube shaft, that is, the distribution and directivity are affected mainly by acoustic frequency and acoustic size of pipe no matter what shape is the leakage hole of pipe. Higher frequencies or larger acoustic size results in stronger directivity and information reflection, that is, the acoustic power is high straight ahead of the leakage source but low at the back. Conversely, lower frequencies or smaller acoustic size results in weaker directivity, that is, the far field sound power level is almost the same in all directions. High frequency sound wave attenuates more quickly than the low frequency sound wave.

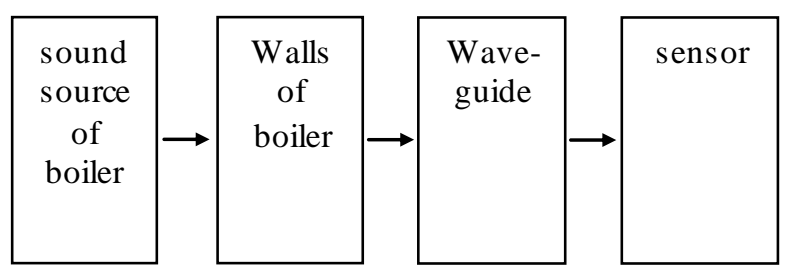

Figure 1. The process of noise propagation in furnace

\section{ACOUSTIC PRINCIPLES OF WAVEGUIDES}

Theoretically, multiply microphone can be applied to realize the monitoring and precise location of the leakage. However, the microphone cannot be installed in the furnace to receive the acoustic signal because of the high temperature. As a result, waveguides need to be applied to collect the acoustic signals and send them to the furnace wall where the temperature is low and is more suitable to install sensors. The design of the waveguides is very crucial because its shape, size, material and state of sound source will affect the sound transmission.

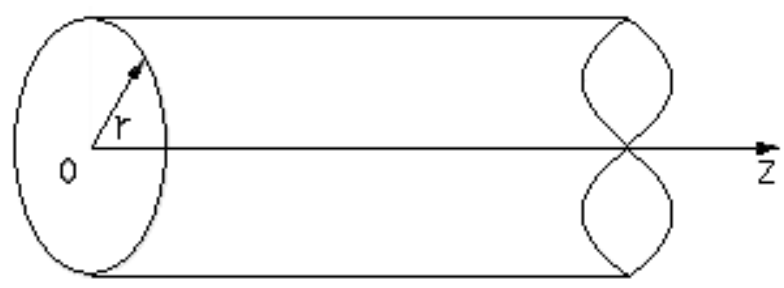

Figure 2. Schematic diagram of microphone

As shown in Fig. 2 for cylindrical waveguide, in tube acoustic equation is described by cylindrical coordinate system, in which $r$ is radial coordinate, $\theta$ is polar Angle and $\mathrm{z}$ is tube axis.

The sound pressure wave equation of cylindrical tube is:

$\frac{\partial^{2} p}{\partial x^{2}}+\frac{\partial^{2} p}{\partial y^{2}}+\frac{\partial^{2} p}{\partial z^{2}}=\frac{1}{c_{0}} \frac{\partial^{2} p}{\partial t^{2}}$

The Laplacian under cylindrical coordinate system is:

$\nabla^{2}=\frac{1}{r} \frac{\partial}{\partial r}\left(r \frac{\partial}{\partial r}\right)+\frac{1}{r^{2}} \frac{\partial^{2}}{\partial \theta^{2}}+\frac{\partial^{2}}{\partial z^{2}}$ 
So the formula (2) transformation is:

$$
\frac{1}{r} \frac{\partial}{\partial r}\left(r \frac{\partial p}{\partial r}\right)+\frac{1}{r^{2}} \frac{\partial^{2} p}{\partial \theta^{2}}+\frac{\partial^{2} p}{\partial z^{2}}=\frac{1}{c_{0}} \frac{\partial^{2} p}{\partial t^{2}}
$$

The detailed derivation is shown in reference $[6,7]$, and the sound pressure in the cylindrical tube is obtained as follows:

$$
P_{m n}=A_{m n} \cos \left(m \theta-\varphi_{m}\right) J_{m}\left(K_{m m} r\right) e^{j\left(\omega t-k_{z} z\right)}
$$

In the formula, $\mathrm{m}$ and $\mathrm{n}$ are non-negative integer;

$\mathrm{A}$ is the acoustic pressure amplitude (constant);

$\theta$ is the angle of wave propagation direction and the $\mathrm{x}$ axis;

$\varphi m$ is the initial angle of sound wave;

$\omega$ is the circular frequency of sound wave;

$\mathrm{t}$ is time;

$\mathrm{kz}$ is constant; Kmmr.

$\mathrm{Jm}(\mathrm{Kmmr})$ is $\mathrm{m}$ order Bessel function of variable

In equation (4), Pmn is the particular solution, which shows a possible acoustic wave in the waveguide that spreads along the $\mathrm{z}$ axis waveguide of the propagation of acoustic waves along the $\mathrm{z}$ axis direction, that is, the cylindrical waveguide $(\mathrm{m}, \mathrm{n})$ in the normal mode. When $\mathrm{m}$ $=0$, for example, $\mathrm{n}=0$ sound waves called $(0,0)$ wave and the sound pressure is expressed as:

$$
P_{00}=A_{00} e^{j\left(\omega t-k_{z} z\right)}
$$

Equation $(5)$ is the $(0,0)$ wave that only spreads along $\mathrm{z}$ axis and where sound pressure amplitude A00 and particle velocity amplitude are consistent. To differentiate various waves in waveguide, $(0,0)$ wave is called the main wave, and $(m, n)$ waves are called higher order waves when $m$ and $n$ are not zeros [5].

As for specific waveguide, the normal frequency fmn can be defined as:

$$
f_{m n}=k_{m n} \frac{c_{0}}{2 \pi a}
$$

In the formula, $\mathrm{Kmn}$ is a group of constant that are soluble, $\mathrm{a}$ is the waveguide radius, and $\mathrm{c} 0$ is sound velocity.

In the waveguide, the condition on which the sound spread along $\mathrm{Z}$ axis is: $\mathrm{f}>\mathrm{fmn}$, that is, only when the radiated frequency of acoustic source is higher than certain normal frequency in waveguide fmn, the corresponding (m, n) wave can be radiated. Otherwise, none of the higher order mode wave will occur in the waveguide because the normal frequency of $(0,0)$ plane wave $\mathrm{f} 00=0$, and any frequency component is higher than zero when there is acoustic source. In other words, acoustic source can radiate $(0,0)$ plane wave that spreads along $Z$ axis.

Therefore, the lowest normal frequency is defined as cutoff frequency of waveguide. For certain waveguide, the cutoff frequency is fixed. Therefore, if all the frequency components are lower than it, there is only one $(0,0)$ plane wave that can spread in the waveguide.

The cutoff frequency of cylindrical wave guide fc [8] is :

$$
f_{c}=f_{10}=k_{10} \frac{c_{0}}{2 \pi a}=1.841 \frac{c_{0}}{2 \pi a}
$$

If more $(m, n)$ waves are available rather than the one that spreads along $\mathrm{z}$ axis, the frequency components of acoustic source must be higher than cutoff frequency to produce stronger noise signal and to increase the sensibility and reliability of signal acquisition system.

\section{SOUND FIELD SIMULATION AND SOUND RAY TRACKING}

\section{A. Setting up Furnace Model}
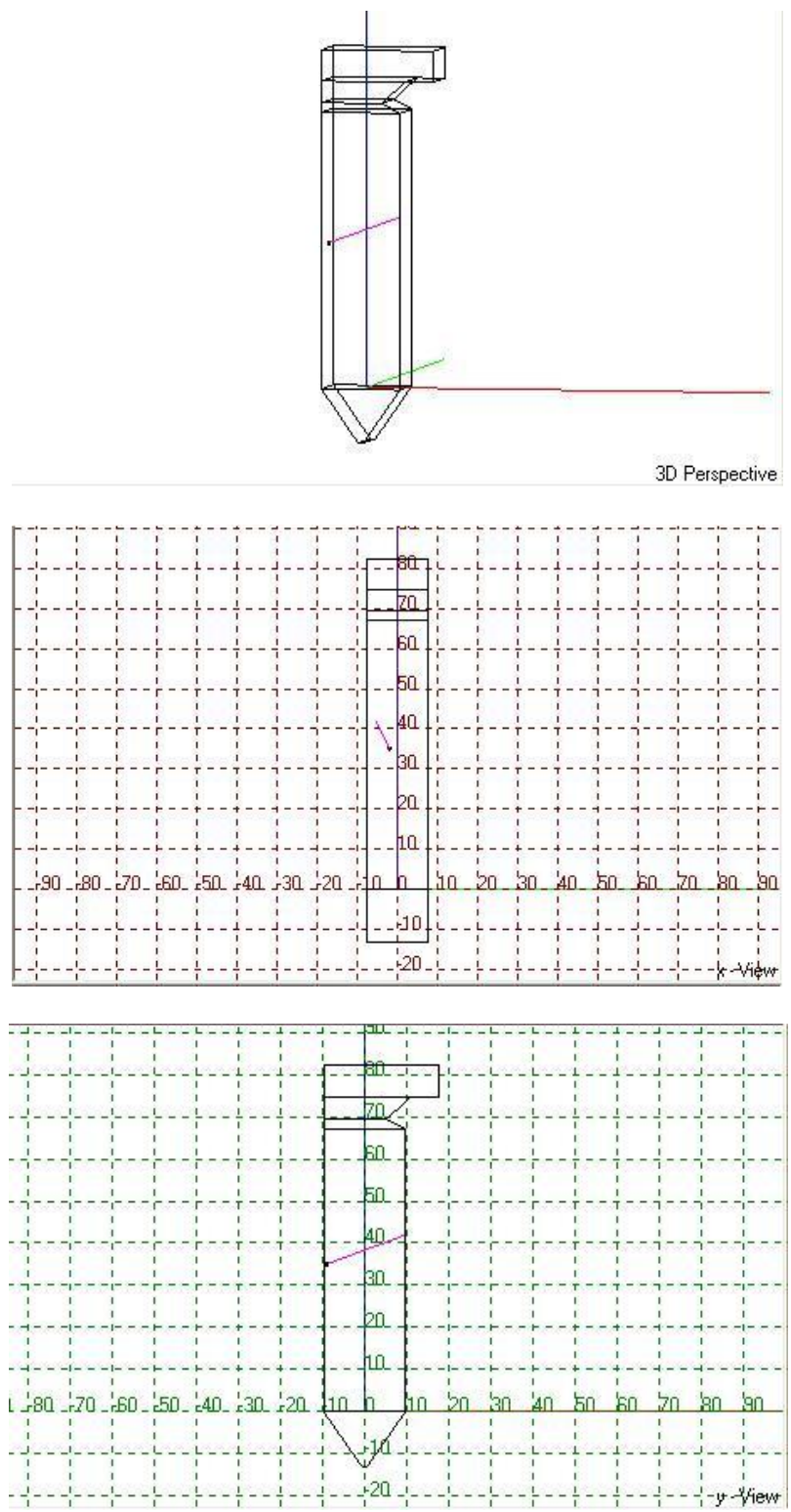


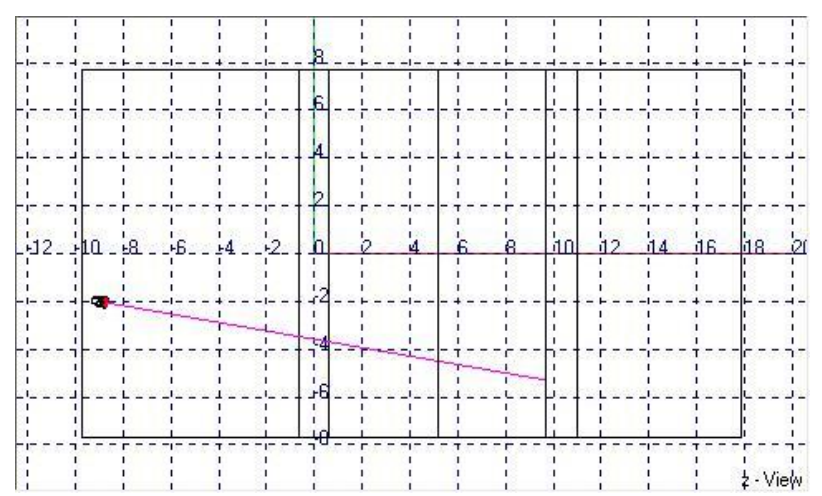

Figure 3. Schematic diagram of furnace model and its view in the $x$ direction, $\mathrm{y}$ direction, $\mathrm{z}$ direction

By employing the EASE software, the boiler simulation model has been established based on the actual size of $660 \mathrm{MW}$ supercritical boiler furnace. The material of the furnace is steel and SP215-62 WAY PAS loudspeaker is employed as leakage source. The origin of coordinates is located in the graphic furnace center, and horizontal position is located in the above the discharge port. Coordinate of leakage source is $(x, y, z)=(-9,-2,35)$. To observe the sound transmission more clearly, certain directivity is set, that is, the angle between leakage source and horizontal level is $20^{\circ}$, and the angle between leakage source and vertical level is $80^{\circ}$, which is shown in Fig. 3 .

\section{B. Transmission Process of Leakage Sound}

The template is used to format your paper and style the text. All margins, column widths, line spaces, and text fonts are prescribed; please do not alter them. You may note peculiarities. For example, the head margin in this template measures proportionately more than is customary. This measurement and others are deliberate, using specifications that anticipate your paper as one part of the entire proceedings, and not as an independent document. Please do not revise any of the current designations.

The establishment of furnace model enables us to observe the transmission process directly. Normally, the process is shown in Fig. 4 when the number of reflection is 4.

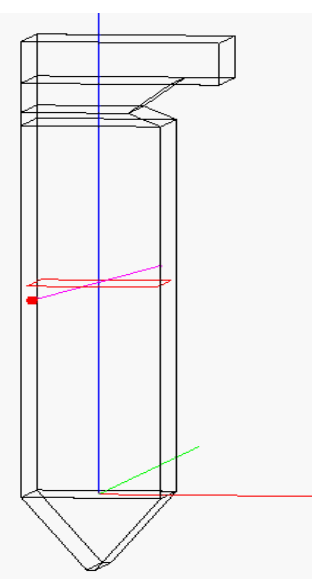

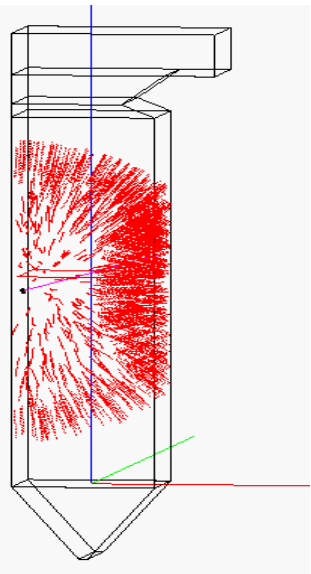

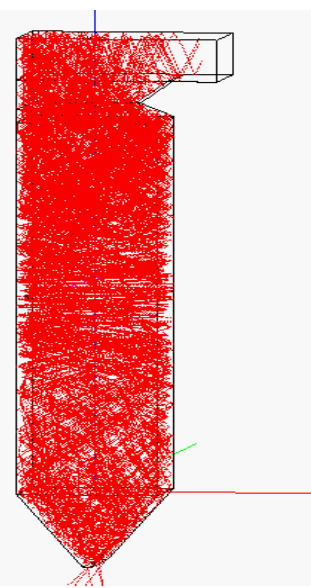

Figure 4. Propagation process of Leakage sound ray

\section{C. leakage sound pressure level analysis}
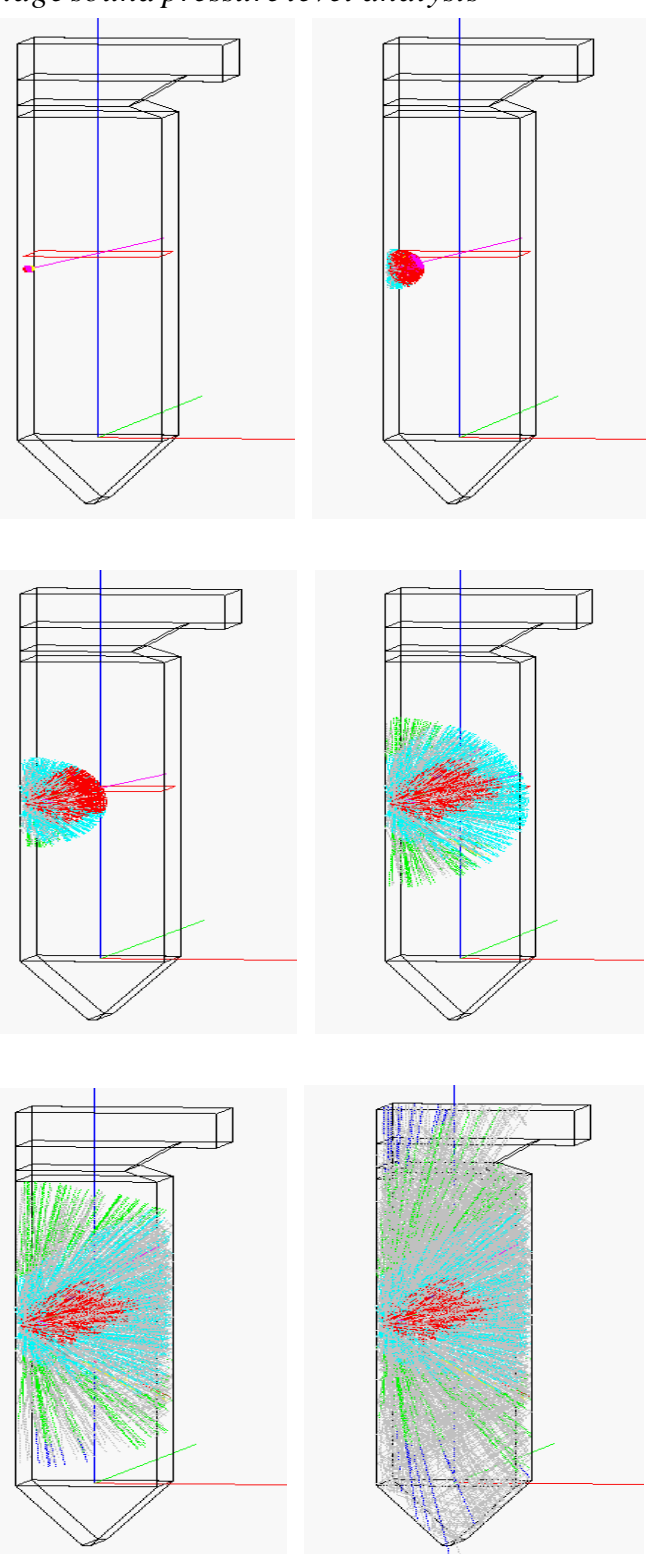

Figure 5. SPL changes of Leakage sound 
The attenuation of leakage sound can be clearly reflected in Fig. 5, in which different colors indicate different sound pressure levels. Precisely, red line represents the sound pressure level of $110 \mathrm{~dB}$ at the leakage sound source, while blue line represents the level of $85 \mathrm{~dB}$ when the sound reaches the opposite side of the furnace [9-11].

\section{SUMMARY}

The leakage sound transmission model is established in this paper with the characteristic of employing waveguides to collect sound signal because the high temperature doesn't allow any sensor in the furnace. Firstly, the acoustic principles have been analyzed theoretically based on the field distribution and directivity characteristics when leakage happens in the boiler furnace. Then the transmission model has been established to make the sound field simulation and line tracking. Lastly, the variation of sound pressure level has been studied to prove the attenuation law.

\section{REFERENCES}

[1] Freund J B. Jet noise physics and modeling using first principles simulations, NASA/CR-2003-212123 [R]. Urbana, Illinois: University of Illinois, Urbana-Champaign, 2003.

[2] Gen-shan Jiang, Lin Wang, Peng Tong, et, al. A Method of Acoustic Detection and Location for Boiler Tube Leakage Employing Cross-Correlation Algorithm [C]. The 10th Western Pacific Acoust ic Conference, September 7 9, 2009, Beijing, China.
[3] An Liansuo, Jiang Genshan, Wang Songling, Yang Kun, A Study on the Trait Value of the Sound Fields Radiated from a Round Leaky Hole on the Heat Exchanger Tube Wall, Proceedings of the CSEE, 2003, 23(5): 144-148.

[4] Jiang Genshan. Characteristics Research on Boiler Pipe Leakage Acoustic Behavior [D]. North China Electric Power University, Baoding, 2006.

[5] Du Gonghuan, Zhu Zhemin, Gong Xiufen. Acoustics Foundation [M]. Nanjing: Nanjing university press, 2001

[6] Ding Sheng. The Leakage Monitoring System for Power Plant Boiler Tubes Based on Acoustic Emission Technology [D]. North China Electric Power University, Baoding, 2005.

[7] Yang Wenze. Experimental research on acoustic wave propagation characteristics in gas-solid two-phase flow medium [D]. North China Electric Power University, Baoding, 2008.

[8] Wang Lin. Researches on the Detection and Location Method for Boiler Tube Leakage Basing Acoustic Radiation Technology [D]. North China Electric Power University, Baoding, 2010.

[9] Tong Peng. Research on Acoustic Monitoring and Location of Boiler Tubes Leakage Based on Information Fusion [D]. North China Electric Power University, Baoding, 2012.

[10] Peng Tong, Liansuo An and Genshan Jiang. Research on Acoustic Character Of Jet Noise Of Boiler Tubes Leakage [C]. 2010 AsiaPacific Power and Energy Engineering Conference, March 28 31, 2010, Chengdu, China.

[11] Lian-suo An, Peng Tong and Gen-shan Jiang. Research on Weak Leak Detection of Boiler Tubes under Strong Background Noise[C], 2011 International Conference on Advanced Engineering Materials and Technology, July 29 31, 2011, Sanya, China. 